\title{
Mass Transfer Through Vapour-Liquid Interfaces: A Molecular Dynamics Simulation Study
}

Simon Stephan, Dominik Schaefer, Kai Langenbach and Hans Hasse

Laboratory of Engineering Thermodynamics (LTD), TU Kaiserslautern, 67663 Kaiserslautern, Germany

\begin{abstract}
ARTICLE HISTORY
Compiled Monday $24^{\text {th }}$ August, 2020

ABSTRACT

A quasi-stationary molecular dynamics simulation method for studying mass transfer through vapourliquid interfaces of mixtures driven by gradients of the chemical potential based on the dual control volume (DCV) method is described and tested. The rectangular simulation volume contains three bulk domains: a liquid domain in middle with vapour on each side such that there are two vapour-liquid interfaces. The mass flux is generated by prescribing the chemical potential in control volumes in the vapour domains close to the outer boundary of the simulation volume. The simulation method was applied for studies of two binary Lennard-Jones mixtures: one in which a strong enrichment of the lowboiling component at the vapour-liquid interface is observed and another in which there is practically no enrichment. The two mixtures differ only in the dispersive interactions; their bulk diffusion coefficients are similar. Furthermore, the prescribed chemical potential difference was the same in all simulations. Nevertheless, important differences in the mass flux of the low-boiling component were observed for the two mixtures at all studied temperatures which might be related to the enrichment at the interfaces.
\end{abstract}

\section{KEYWORDS}

vapour-liquid interface, mass transfer, binary mixture, Lennard-Jones fluid, molecular dynamics, enrichment

\section{Introduction}

The mass transfer through fluid interfaces of mixtures is of fundamental importance in many technical processes, for example in chemical industry. The most important of fluid separation techniques, distillation and absorption, are based on the mass transfer of components through vapour-liquid interfaces. The driving force in these processes is a gradient in the chemical potential, whereas temperature and pressure gradients usually play only a minor role. It is astonishing that up to now no methods 
for studying the fundamentals of mass transfer through vapour-liquid interfaces by molecular simulations under these conditions have been described in the literature. We have therefore developed such a method; it is presented here together with first results, in which the method was applied to study a relevant current problem: the influence of the enrichment of components on the mass transfer through vapour-liquid interfaces.

Some related simulation methods have been described previously in the literature. The heat and mass transfer through vapor-liquid interfaces during evaporation and condensation has been studied extensively in the literature using molecular simulation [1-19] or mesoscopic models such as density functional theory $[8,13,17,19-23]$. Most of these studies consider a temperature gradient as the driving force of the heat and mass transfer. Furthermore, in most of these studies, only pure components are considered.

Diffusion near vapour-liquid interfaces is known to be anisotropic. The available studies on this subject deal mostly with self-diffusion [24-27]. Interestingly, the diffusion parallel to the interface has been investigated more often than the diffusion perpendicular [27-29] to the interface, which is relevant for the mass transfer in fluid separation techniques.

Nagl et al. [30] recently reported a combined theoretical-experimental investigation of the mass transfer through liquid-liquid interfaces. Also Braga et al. [31] investigate diffusion at liquid-liquid interfaces. The free energy barrier of particles crossing a vapour-liquid interface has been studied by Braga et al. [28] and Garrett et al. [32]. The build up of the interfacial excess at vapor-liquid interfaces of binary mixtures in a relaxation process has been studied recently by Baidakov et al. [33, 34].

The simulation method employed in the present work is based on the so-called dual control volume (DCV) method, which has been applied several times for studying diffusion in crystals, membranes, and pores [35-42], but to the best of our knowledge not for mass transfer across vapor-liquid interfaces. In the DCV method, the chemical potential in two sub-sections of the simulation volume is prescribed by a Monte Carlo algorithm. Particles are then inserted in one sub-section and removed from the other, causing a mass flux. We adapt the basic idea of the dual control volume method [37, 41, 43-45] to induce a mass flux through vapour-liquid interfaces driven by a gradient of the chemical potential. This approach was applied in the present work on a rectangular simulation volume in which there is a liquid slab in the middle with a vapour slab on each side. In each of the vapour domains, there is a control volume near the outer boundary of the simulation volume. The chemical potential is prescribed such that there is a mass transfer through the two vapour-liquid interfaces. The developed simulation scenario is tested using simple Lennard-Jones model mixtures. In these tests, we address a current topic: the influence of the enrichment of components at the vapour-liquid interface on the mass transfer [46].

Results from equilibrium molecular thermodynamics show an enrichment of low-boiling components 
at vapour-liquid interfaces for many mixtures [46-65]. This enrichment is predicted consistently by molecular simulations, i.e. Monte Carlo (MC) or molecular dynamics (MD) simulations, as well as density gradient theory (DGT) $[46,47,52,53,66-68]$. Since the enrichment is a nanoscopic effect at fluctuating fluid interfaces, currently no experimental methods are available to study it directly. The enrichment of components at fluid interfaces of mixtures is believed to influence the mass transfer through fluid interfaces [30, 47, 49, 59, 69-73], but a proof is still lacking.

The new simulation scenario is applied on two different binary Lennard-Jones mixtures. The equilibrium interfacial and bulk properties of the Lennard-Jones mixtures used in the present work have been studied systematically in earlier works of our group [47, 48, 50, 51]. For the first mixture, the low-boiling component exhibits a strong enrichment at the interface; for the second, the low-boiling component exhibits no enrichment at the interface. Non-equilibrium Simulations with both mixtures are performed at four temperatures in the present work.

Additionally, the homogeneous bulk phase diffusion coefficients were determined for both mixtures at the studied temperatures using Green-Kubo simulations for the liquid phase and the ChapmanEnskog theory for the vapour phase. The information on the bulk diffusion coefficients is prerequisite for the modelling of the mass transfer with continuum methods [74, 75] and a comparison of the results with those from the molecular simulations. The development of such continuum models was not in the scope of the present study.

This paper is organised as follows: first, the simulation method is introduced. Then the two mixtures are defined and described. The simulation results for the two mixtures are then presented and compared; finally, conclusions are drawn.

\section{Molecular Simulations}

\subsection{Overview of the Simulation Method}

In the present section, the molecular simulation method for studying the mass transfer at vapourliquid interfaces driven by a gradient in the chemical potential is described in a general way. The actual settings that were used for the simulations in the present work are reported below.

The simulation scenario is schematically shown in Fig. 1. It contains a liquid domain in the middle and two vapour domains on each side. Each of the vapour domains contains a control volume (labelled $\mathrm{CV}+$ and $\mathrm{CV}-$ ), in which the chemical potential is prescribed such that the chemical potential difference causes a mass flux in $+z$-direction, which passes through both vapour-liquid interfaces. This mass flux is the most important observable in the simulation. Periodic boundary conditions are applied in $x$ - and $y$-direction perpendicular to the interfaces. In $z$-direction, the simulation volume is confined by 
soft repulsive walls.

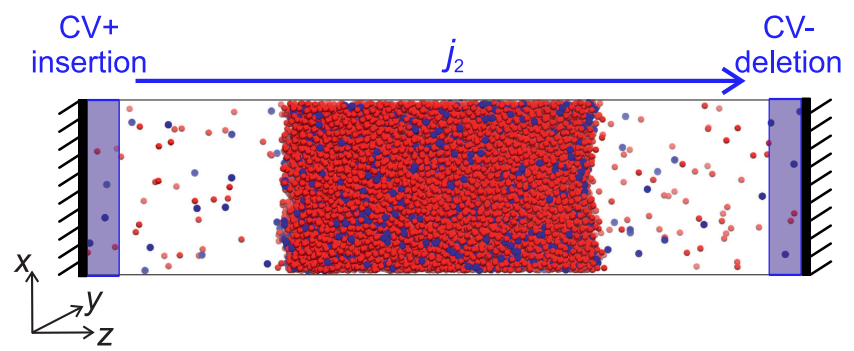

Figure 1. Scheme of the simulation set-up used in the present work for studying the mass transfer through vapour-liquid interfaces in binary mixtures. Red spheres indicate the high-boiling component 1 and blue spheres the low-boiling component 2 . A liquid domain in the middle is surrounded by two vapour domains. The simulation box has periodic boundaries in $x$ - and $y$-direction and soft repulsive walls in $z$-direction. The chemical potential of component 2 is adjusted to prescribed values in the control volumes $\mathrm{CV}+$ and $\mathrm{CV}-$ (shaded blue) by a Monte-Carlo algorithm. The resulting flux $j_{2}$ of component 2 is measured.

As described in more detail in the next section, binary Lennard-Jones mixtures were studied in the present work for testing the simulation scenario. The high-boiling component is component 1, the low-boiling component is component 2. The chemical potential difference was only set for component 2. No action on component 1 was taken in the control volumes. The resulting flux $j_{2}$ of component 2 is defined here as the mean number of particles per time passing through a plane perpendicular to the $z$-axis. All fluxes are reported in the fixed laboratory frame. The flux of component $2 j_{2}$ can also be interpreted as a diffusive flux, when component 1 is taken as reference (as the velocity of that component is zero). The use of a number averaged mean velocity as reference for defining the diffusive fluxes is briefly discussed in the Appendix.

Quasi-stationary conditions were established before the sampling was started. The magnitude of the chemical potential difference of component 2 was chosen such that the resulting flux of component 2 was low, but still reasonably measurable. The resulting response is described well by linear theory.

The simulation was specified as follows: the temperature $T$ was prescribed in the entire simulation volume using velocity-scaling. As the particle velocity from the Brownian movements is much larger than the averaged component velocity that causes the flux, the thermostat was simply applied to the actual velocities of all particles without subtracting the average component velocity. The error induced by this simplification is negligible. The total volume of the simulation box $V$ was constant. Due to the boundary conditions, the number of particles of component $1 N_{1}$ was also constant. Particles of component 2 were inserted into the control volume CV+ and removed from the control volume CVby prescribing the number for the chemical potential of component 2 in the two control volumes $\mu_{2}^{\mathrm{CV}+}$ and $\mu_{2}^{\mathrm{CV}-}$. This was achieved by a grand canonical Monte Carlo algorithm [43-45].

The simulation was carried out as follows: the initialisation and an equilibration were carried out in the NVT ensemble - as it is done in direct vapour-liquid equilibrium (VLE) simulations. After that, the control volumes, which are placed next to the soft repulsive walls, were switched on. With all 
settings active, the simulation was run until the mass flux of component 2 through the simulation box was stationary; then, the sampling was started.

The primary measured observable during the production phase is the flux of component $2 j_{2}$. It is measured by monitoring the number of particles of component 2 that are inserted in the control volume $\mathrm{CV}+$ and those that are deleted in the control volume CV-. These numbers are equal - within their fluctuation band-width - in the stationary sampling phase. The area that is used for calculating the flux is the geometric cross section of the simulation box perpendicular to the $z$-direction. Besides the flux $j_{2}$, also profiles of different variables depending on the $z$-position in the simulation box were sampled. For that purpose, the box was divided into bins in $z$-direction, in which the number densities of both components $\rho_{1}(z)$ and $\rho_{2}(z)$ and the diagonal components of the pressure tensor were measured.

The pressure in the bulk domains in the simulation box is not constant in $z$-direction. This can be understood as a consequence of prescribing the temperature and imposing a chemical potential gradient that controls the concentration profiles. The pressure gradient and the flux of the particles of component 2 act in such a way that the momentum balance is practically fulfilled in any sub-volume in the simulation box, i.e. no measures had to be taken to stabilise the position of the liquid slab in the centre of the simulation volume.

The simulations with the outlined scenario pose several challenges. Unfavourable choices for the difference of the chemical potential between both control volumes can yield nucleation of droplets in the vapour domain, separation of the liquid film, movements of the liquid film during simulations, and exceedingly long simulation times to reach a quasi-stationary state. However, by choosing suitable settings, these problems can be circumvented. Further details on the simulation method are given in the Supplementary Material.

\subsection{Studied Lennard-Jones Mixtures}

The simulation method introduced in the present work was used to study Lennard-Jones mixtures. The Lennard-Jones fluid is one of the most frequently studied model fluids [76, 77] as it is computationally relatively inexpensive but still yields a realistic description of simple fluids. It is therefore often used to test new methods in molecular simulations and fluid state theory [67, 78-82]. In the present work, the Lennard-Jones truncated and shifted (LJTS) potential with a cut-off radius of $2.5 \sigma$ is used:

$$
\begin{array}{r}
u_{\mathrm{LJ}}(r)=4 \varepsilon\left[\left(\frac{\sigma}{r}\right)^{12}-\left(\frac{\sigma}{r}\right)^{6}\right] \\
u_{\mathrm{LJTS}}(r)= \begin{cases}u_{\mathrm{LJ}}(r)-u_{\mathrm{LJ}}\left(r_{\mathrm{c}}\right) & r \leq r_{\mathrm{c}} \\
0 & r>r_{\mathrm{c}},\end{cases}
\end{array}
$$


where $u_{\mathrm{LJ}}$ is the full Lennard-Jones potential, $\varepsilon$ and $\sigma$ are the energy and size parameter, respectively. The distance between two particles is $r$. The truncation radius $r_{\mathrm{c}}$ of the potential is $2.5 \sigma$ throughout the present work. No long range corrections are required in the simulations.

Two binary LJTS systems (named mixture A and B in the following) were studied in this work. The high-boiling component 1 is the same in both mixtures. The low-boiling character of component 2 was obtained by decreasing its dispersion energy $\varepsilon_{2}$ with respect to $\varepsilon_{1}$ of component 1 . The size parameter and the mass of the components 1 and 2 are the same for both mixtures, i.e. $\sigma_{1}=\sigma_{2}$ and $m_{1}=m_{2}$.

All physical properties are conveniently reduced in the present work using the Lennard-Jones potential parameters of the high-boiling component $\varepsilon_{1}, \sigma_{1}$ and the molecular mass $m_{1}$, as well as the Boltzmann constant $k_{\mathrm{B}}[83]$.

The cross-interactions between unlike particles were modelled using the modified Lorentz-Berthelot combination rules $[84,85]$

$$
\begin{aligned}
\sigma_{i j} & =\frac{\sigma_{i}+\sigma_{j}}{2}, \\
\varepsilon_{i j} & =\xi \sqrt{\varepsilon_{i} \varepsilon_{j}}
\end{aligned}
$$

where the single index $i$ and $j$ stands for the interaction of two particles of the same component and the double index $i j$ for the cross interaction between different components.

The two binary LJTS mixtures investigated here are:

- Mixture A (asymmetric wide-boiling mixture): $\varepsilon_{2} / \varepsilon_{1}=0.6$ and $\xi=0.85$,

- Mixture B (ideal mixture): $\varepsilon_{2} / \varepsilon_{1}=0.9$ and $\xi=1$.

Both bulk and interfacial equilibrium properties of these two mixtures have been investigated systematically in previous works of our group [47, 48, 50, 51]. Mixture B is essentially an ideal mixture in the sense of Raoult's law, whereas mixture A is highly asymmetric and strongly non-ideal. It exhibits a vapour-liquid-liquid equilibrium at low temperatures [50]. In mixture $\mathrm{B}$, the low-boiling component 2 exhibits practically no enrichment at the vapour-liquid interface in the entire composition and temperature range, whereas in mixture A component 2 exhibits a large enrichment [50]. This enrichment is known to increase with decreasing temperature and decreasing mole fraction of the low-boiling component. There is no enrichment of the high-boiling component 1 at the vapour-liquid interface, neither for mixture A nor for mixture B [50].

The mass transfer through the vapour-liquid interfaces was studied with the new simulation method for both mixture $\mathrm{A}$ and $\mathrm{B}$ at four temperatures: $T / \varepsilon k_{\mathrm{B}}^{-1}=0.66,0.715,0.77,0.825$. For mixture A, the component 2 is supercritical at all investigated temperatures, while for mixture $\mathrm{B}$, the component 2 is 
subcritical at all investigated temperatures.

\subsection{Simulation Details}

We will first explain the principles and then discuss their realisation. Let us start by considering an equilibrium situation (or, equivalently, assume that only a negligible gradient in the chemical potential of component 2 was applied). Then, after the equilibration, a vapour-liquid equilibrium would be established in the simulation. Let us first consider this vapour-liquid equilibrium in intensive variables. In our simulations, it is specified by the temperature $T$ and the chemical potential of component 2 $\mu_{2}$. Furthermore, the number of particles of component $1 N_{1}$ as well as the simulation volume $V$ are set $\left(N_{2}\right.$ is not set and will adjust itself to meet the specification of $\left.\mu_{2}\right)$. Hence, such a simulation can be characterised as an $N_{1}, V, T, \mu_{2}$-simulation. These settings fully determine the vapour-liquid equilibrium in the simulation box, including the amounts of the vapour and the liquid phase and the corresponding compositions. Starting from this equilibrium, the finite gradient of the chemical potential of component $2 \mu_{2}$ is imposed by increasing $\mu_{2}$ in one control volume $\mathrm{CV}+$ by $\delta \mu_{2}$ and decreasing it by the same amount in the other control volume $\mathrm{CV}-$. The resulting quasi-stationary state is then sampled in the simulation, which is near the equilibrium state described above for small $\delta \mu_{2}$. For a binary mixture at constant temperature, the gradients of the chemical potentials of both components and the pressure are coupled by the Gibbs-Duhem equation. As a result of the imposed gradient of the chemical potential of component 2 , in general, also the gradients of the chemical potential of component 1 and the pressure are non-zero. The resulting diffusive and convective mass flux of component 1 cancel each other, as a consequence of the employed boundary conditions that do not allow a net flux of component 1 in the stationary simulation. The new simulation scenario is based on these principles; but the realisation involves additional considerations, that are discussed now.

The rectangular simulation volume $V$ used in the present work has a quadratic cross section in $x$ and $y$-direction with a side length $a$. The length of the simulation volume in $z$-direction was always $b=170 \sigma$. The length $a$ was $21.1 \sigma$ in the simulations with mixture A and $22 \sigma$ in the simulations with mixture B. The settings were chosen such that the thickness of the liquid domain (in $z$-direction) was about $40 \sigma$ and the thickness of the vapour domain was $65 \sigma$ each, cf. Fig. 2. The maximal range of the soft repulsive wall potential was $5 \sigma$ and the thickness of the control volumes was $10 \sigma$, such that each of the two undisturbed bulk vapour domains has a thickness of $50 \sigma$, when these regions are excluded. The total particle number $N_{1}+N_{2}$ was about 16,000 in all simulations. The selection of the actual choices for the initial densities and compositions was supported by preliminary calculations of the phase equilibrium using the PeTS EOS [47, 86].

The soft repulsion of the walls acts on both components 1 and 2 and is modelled by half a harmonic 


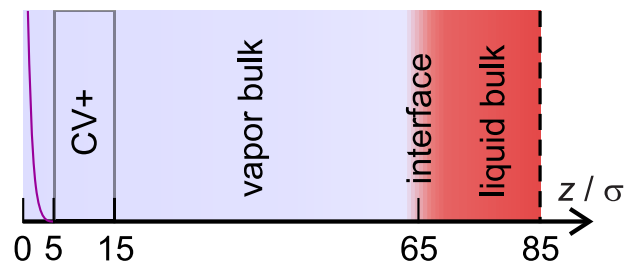

Figure 2. Geometric parameters of the scenario used in the present simulations. Only one half of the geometrically symmetric simulation box is shown; the symmetry plane is indicated by the dashed line. The cross section of the simulation box in the $x, y$ plane was quadratic (edge length was $21.1 \sigma$ for mixture A simulations and $22 \sigma$ for the mixture B). The purple parable indicates the soft repulsive wall. The control volume is the grey line.

potential which starts to become effective at a distance of $5 \sigma$ from the end of the simulation box, as indicated in Fig. 2. At closer distances, a repulsive force acts on the particle that rises linearly with a force constant of $k=20 \varepsilon \sigma^{-2}$.

In the left control volume CV+ (cf. Fig. 1) particles of component 2 were inserted; and in the right control volume $\mathrm{CV}$ - particles of component 2 were removed from the simulation box. The choice of the chemical potentials of component $2 \mu_{2}$ in the control volumes is not trivial. Basically, $\mu_{2}$, as determined in the NVT equilibrium simulations was increased by $\delta \mu_{2}$ in the control volume $\mathrm{CV}+$ and decreased by the same $\delta \mu_{2}$ in the control volume $\mathrm{CV}-$. The difference of the chemical potentials between the two control volumes was the same in all simulations and was $\Delta \mu_{2}=0.5 \varepsilon$. Preliminary simulations showed that this is enough to ensure a reliable determination of the flux of component 2 , but still well in the linear regime. It turns out that this value chosen based on computational considerations lies in the same range as the chemical potential differences that are common in chemical engineering mass transfer problems [87]. However, the chemical potential gradient, which results from this choice, is much larger than that in common chemical engineering problems, as the distance of the control volumes is of the order of $10^{-7} \mathrm{~m}$ whereas the boundary layers in chemical engineering mass transfer problems are of the order of $10^{-4} \mathrm{~m}$ [87]. The corresponding difference of $3-4$ orders of magnitude is also found for the fluxes between engineering mass transfer problems and the fluxes obtained from the mass transfer simulations of the present work.

The simulation was carried out as follows: the first phase of the simulation is a pre-run in the NVT ensemble. It is used to establish an equilibrium with a liquid slab in the middle and the two surrounding vapour domains, see Figs. 1 and 2. The initial positions and velocities of the particles were chosen as it is common in direct simulations of phase equilibria; the description given in Ref. [48] applies also here, correspondingly. The time step was $\delta \tau=0.001 \sigma \sqrt{\mathrm{m} / \varepsilon}$ in all simulations; the NVT simulations were carried out for 50,000 time steps. After this, the control volumes in the vapour domains were switched on to evoke the mass flux $j_{2}$ in $z$-direction through the simulation box. The simulations were then run for at least 3,000,000 further time steps, until a stationary mass flux was established. The 
settings for the MC algorithm that was used for this purpose in the control volumes were: 50 Monte Carlo insertion/ removal trials every $40 \mathrm{MD}$ time steps. The insertion and removal were steered by a grand canonical Monte Carlo algorithm, cf. Refs. [43-45] for details.

After a steady state was achieved, the sampling was started and carried out for at least 5,000,000 time steps. The simulation box was divided in 1200 equal bins by a discretisation in the $z$-direction.

The mass flux $j_{2}=\frac{\Delta N_{2}}{\Delta \tau a^{2}}$ was determined from the total number of inserted and removed number of particles during the production time $\Delta \tau$. The cross section of the simulation box is $a^{2}$. The number of inserted and deleted particles $\Delta N_{2}$ was computed from the average of the inserted and deleted particles by the end of the production phase $\tau_{\text {end }}$, i.e. $\Delta N_{2}=\frac{N_{2}^{\mathrm{CV}+}\left(\tau_{\text {end }}\right)+N_{2}^{\mathrm{CV}-}\left(\tau_{\text {end }}\right)}{2}$. In all cases, $N_{2}^{\mathrm{CV}+}\left(\tau_{\text {end }}\right)$ and $N_{2}^{\mathrm{CV}-}\left(\tau_{\text {end }}\right)$ deviate by no more than $10 \%$. Details are given in the Supplementary Material.

The pressure profile and the component density profiles were sampled in these bins. Density and pressure profiles were computed in block averages of 200,000 time steps during the production phase. During the entire simulation time, the box was temperature-controlled by velocity scaling, as discussed in Section 2.1. The velocity associated with the flux of the particles of component 2 was two to three orders of magnitude smaller than the mean thermal motion velocity. The flux $j_{2}$ of component 2 was measured by counting the insertions and deletions of particles of component 2 in the control volumes in the production phase. A detailed discussion of the sampling and the computation of the statistical uncertainties is given in the Supplementary Material.

An inspection of the profiles (see results section) shows, that the gradients of the pressure and the component densities in the bulk domains are moderate. For the discussion, it is helpful to consider the average properties over the three bulk domains, which are labelled here $\mathrm{V}_{\text {left }}$ (vapour left), $\mathrm{L}$ (liquid), and $\mathrm{V}_{\text {right }}$ (vapour right). The density $\rho$, the pressure $p$, and the composition $x_{i}$ were calculated as an average over the respective bulk domain excluding the area close to the interface and close to the control volumes, i.e. keeping a distance of at least $10 \sigma$ to the control volume and to the centre of the interface. The corresponding statistical uncertainties were computed from the standard deviation of the individual bin values.

Furthermore, the enrichment $E_{2}$ of the low-boiling component at the interfaces $[47,53]$ was computed from each density profile from the production phase as

$$
E_{2}=\frac{\max \left(\rho_{2}(z)\right)}{\max \left(\rho_{2}^{\mathrm{L}}, \rho_{2}^{\mathrm{V}}\right)}
$$

Here, $\rho_{2}^{\mathrm{L}}$ and $\rho_{2}^{\mathrm{V}}$ are the densities in the adjoining bulk domains. For each simulation and interface, $E_{2}$ and its uncertainty were computed as the mean value from the block averages and three times their standard deviation, respectively. 
The mass transfer simulations were carried out using the molecular dynamics code $l s 1$ [88].

\subsection{Bulk Diffusion Coefficient Simulations}

Diffusion coefficients were computed for both mixtures in the liquid and the vapour phase. For the liquid phase, molecular simulations were performed at state points slightly above the bubble line. For the vapour phase, diffusion coefficients were determined from the Chapman-Engskog theory [89].

The liquid bulk diffusion coefficients were determined with the molecular simulations code $m s 2$ [90] using the Green-Kubo formalism as in previous work of our group [91-95]. Simulation details are reported in the Supplementary Material.

\section{Results and Discussion}

\subsection{Overview}

Table 1 and 2 report the numeric values for the mass flux $j_{2}$ and the enrichment $E_{2}$ in the mass transfer simulations for all considered temperatures. The numeric values of the average bulk domain state points and the interface positions are reported in the Supplementary Material.

Also the results for the bulk diffusion coefficients are presented in the Supplementary Material. As no unexpected findings were made, they are are not discussed here.

In the following, the results of the mass transfer simulations are discussed, starting with a detailed consideration of a single temperature, before also the results for other temperatures are presented and discussed.

\subsection{Temperature $T=0.715 \varepsilon k_{\mathrm{B}}^{-1}$}

For the simulations at $T=0.715 \varepsilon k_{\mathrm{B}}^{-1}$, the mass flux sampled in the production phase was $j_{2}=$ $0.11 \cdot 10^{-3} \sigma^{-3}(\mathrm{~m} / \varepsilon)^{-0.5}$ for mixture A and $j_{2}=0.26 \cdot 10^{-3} \sigma^{-3}(\mathrm{~m} / \varepsilon)^{-0.5}$ for mixture B (the unit of $j_{2}$ corresponds to the dimension of the number of particles per area and time). Hence, the obtained mass flux in mixture A is smaller than that for the mixture B by more than a factor of two, even though the chemical potential gradient and the bulk phase transport diffusion coefficient of both mixtures are approximately the same - see Supplementary Material for details. In all cases, no net flux of component 1 was observed, in accordance with the chosen simulation settings (see discussion above).

Here, only fluxes in the fixed laboratory frame are discussed. A brief discussion of the corresponding mean directed velocities and the diffusive fluxes in various reference systems is also given in the Appendix. 
Table 1. Numeric values of the mass flux $j_{2}$, the prescribed residual chemical potential in the inserting and deleting control volume $\mu_{2}^{\mathrm{CV}+}$ and $\mu_{2}^{\mathrm{CV}-}$, respectively, and the number of component 1 particles $N_{1}$ in the performed mass transfer simulations for both mixtures $\mathrm{A}$ and $\mathrm{B}$ at all four investigated temperatures. The box size was $170 \sigma$ in $z$-direction in all cases; the $x y$-cross section was a square with the edge length $21.1 \sigma$ for the simulations with mixture A and $22 \sigma$ for the simulations with mixture B.

\begin{tabular}{l|cc|rr|rr|rc}
\hline \hline$T / \varepsilon k_{\mathrm{B}}^{-1}$ & \multicolumn{1}{c}{$j_{2} / 10^{3} \sigma^{-3}(m / \varepsilon)^{-0.5}$} & \multicolumn{1}{c}{$\mu_{2}^{\mathrm{CV}+} / \varepsilon$} & \multicolumn{1}{c}{$\mu_{2}^{\mathrm{CV}-} / \varepsilon$} & \multicolumn{1}{c}{$N_{1}$} \\
& \multicolumn{1}{c}{$\mathrm{A}$} & \multicolumn{1}{c}{$\mathrm{B}$} & \multicolumn{1}{c}{$\mathrm{A}$} & \multicolumn{1}{c}{$\mathrm{B}$} & \multicolumn{1}{c}{$\mathrm{B}$} & \multicolumn{1}{c}{$\mathrm{B}$} \\
\hline 0.66 & $0.06(5)$ & $0.23(1)$ & -0.75 & -2.22 & -0.25 & -1.72 & 12705 & 12447 \\
0.715 & $0.11(5)$ & $0.26(1)$ & -0.8 & -2.4 & -0.3 & -1.9 & 12703 & 12344 \\
0.77 & $0.10(4)$ & $0.21(1)$ & -0.95 & -2.6 & -0.45 & -2.1 & 12642 & 12344 \\
0.825 & $0.19(3)$ & $0.24(1)$ & -1.1 & -2.6 & -0.6 & -2.1 & 12689 & 12347 \\
\hline \hline
\end{tabular}

Fig. 3 - left and middle shows the density and pressure profiles obtained from the mass transfer simulations for mixtures $\mathrm{A}$ and $\mathrm{B}$ at $T=0.715 \varepsilon k_{\mathrm{B}}^{-1}$ during the production phase. The density profiles in Fig. 3 show that the density of the low-boiling component exhibits a gradient $\frac{\partial \rho_{2}}{\partial z}$ in the bulk domains as a response to the gradient of the chemical potential, whereas the total density exhibits no gradient in the bulk domains. Since the diffusion coefficient is lower in the liquid phase than in the vapour phase (see Supplementary Material), the gradient $\frac{\partial \rho_{2}}{\partial z}$ is larger in the liquid bulk domain than in the vapour bulk. This holds for both mixtures.
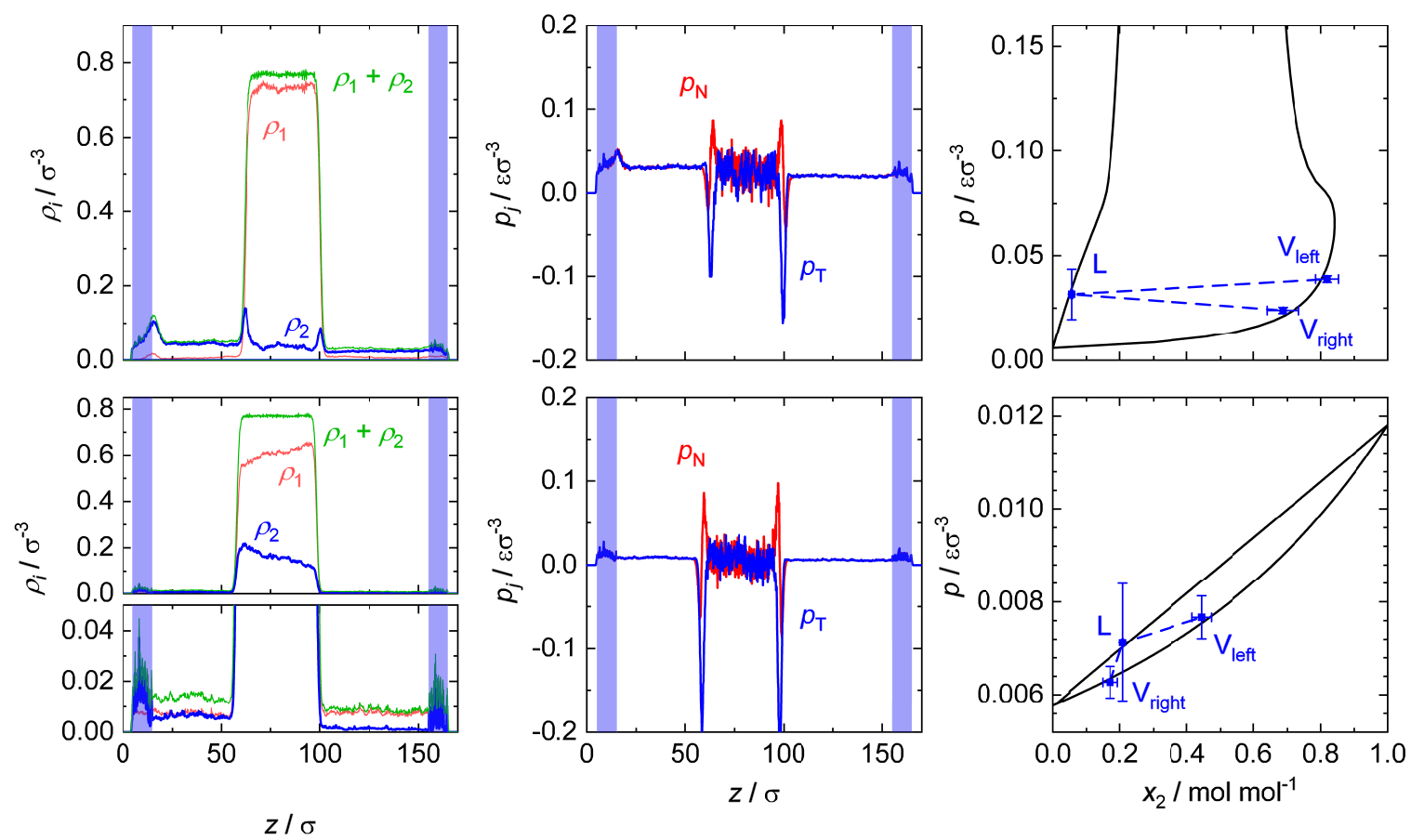

Figure 3. Density profiles (left), pressure profiles (middle), and averaged thermodynamic state points in the bulk domains (right) for the mixtures A (top) and B (bottom) obtained in mass transfer simulations at $T=0.715 \varepsilon k_{\mathrm{B}}^{-1}$. The lower part of the split plot (bottom-left panel) is just an expansion of the upper part. The blue shaded area indicates the control volumes. For the pressure profiles, the indices $j=N, T$ indicate the normal and tangential component of the pressure tensor, respectively. The thermodynamic state points indicated as symbols in the right plots were sampled in the three bulk domains during the production phase of the simulations. They are compared to results for the vapour-liquid equilibrium computed with the LJTS EOS [47, 86] (lines). The density and pressure profiles are from one arbitrarily selected block average during the production phase, the state points depicted in the $p-x$ diagrams (right) are the average of all blocks.

For mixture A, the low-boiling component exhibits a large enrichment at the interface, which is not observed for the mixture B. To the best of our knowledge, this is the first time that the enrichment is 
studied under the influence of a mass transfer. This is in line with results from equilibrium simulations for these mixtures $[47,50]$. The numeric values for the enrichment in mixture $\mathrm{A}$ at the left and right interface are compared in Table 2 with equilibrium results from the literature [50] at the same liquid phase composition. Two values are reported for the enrichment at the right interface. The evaluation of Eq. (5) requires the computation of the larger value of the density of component 2 in the adjoining bulk domains as reference in the denominator. In equilibrium simulations, the choice poses no problem as the bulk is equilibrated. In mass transfer simulations, however, gradients occur. This introduces an ambiguity in the choice of the bulk value: it could be chosen as the average value of the domain, or be selected as values at the foot of the peak. For the left peak, this makes no difference, the reference is always the vapour domain and there is no substantial difference between the average vapour density and that at the foot of the peak. For the right peak, this is different. When the average bulk densities are used for the evaluation of Eq. (5), the reference is the liquid density; when the values at the foot of the peak are used, the reference is the vapour density (for which the value at the foot of the peak is again basically the same as the average value). Hence, we report two values for the right peak.

Table 2. Numeric values of the enrichment of the low-boiling component at the interface for mixture A at all four investigated temperatures. The column $x_{2}^{\mathrm{L}}$ indicates the mole fraction of component 2 in the liquid bulk domain L sampled during the mass transfer simulations. The columns $E_{2}^{\text {left }}$ and $E_{2}^{\text {right }}$ indicate the enrichment at the left and right interface, respectively. For the right interface, V and L indicate the evaluation of Eq. (5) using the vapour or liquid domain as reference, respectively (details are given in the text). $E_{2}^{\mathrm{eq}}$ indicates the equilibrium enrichment at the corresponding temperature and liquid phase composition taken from Ref. [50].

\begin{tabular}{lrcrrc}
\hline \hline$T / \varepsilon k_{\mathrm{B}}^{-1}$ & $x_{2}^{\mathrm{L}} / \mathrm{mol} \mathrm{mol}^{-1}$ & $E_{2}^{\text {left }}$ & $E_{2}^{\text {right,L }}$ & $E_{2}^{\text {right, },}$ & $E_{2}^{\text {eq }}$ \\
\hline 0.66 & $0.057(5)$ & $4.4(2)$ & $3.2(3)$ & $5.4(4)$ & $4.0(3)$ \\
0.715 & $0.056(3)$ & $3.2(2)$ & $2.6(2)$ & $3.7(4)$ & $3.1(1)$ \\
0.77 & $0.058(4)$ & $2.5(1)$ & $1.9(2)$ & $2.9(2)$ & $2.5(1)$ \\
0.825 & $0.055(3)$ & $2.1(1)$ & $1.6(2)$ & $2.2(2)$ & $1.9(1)$ \\
\hline \hline
\end{tabular}

As a reference, we have also included results in Table 2 for the enrichment obtained for the same mixture from vapour-liquid equilibrium simulations at the same temperature and the same composition of the liquid bulk domain. The results are also illustrated in Fig. 4, from which it can be seen that the results for the left interface that were obtained in the mass transfer simulations agree very well with those from equilibrium simulations. For the right interface, depending on the choice of the reference, the results lie below or above the corresponding equilibrium values. Overall, it can be concluded, that the values for the enrichment $E_{2}$ do not change strongly when mass transfer occurs, at least for moderate gradients of the chemical potential, as they were applied here.

No enrichment is found for mixture B, i.e. $E_{2}=1$ within the numeric uncertainty in all experiments, cf. component density profiles in the Supplementary Material. This finding is in line with the findings from the equilibrium simulations [47, 50].

For both mixtures $\mathrm{A}$ and $\mathrm{B}$, the average state points computed for the three bulk domains $\mathrm{V}_{\text {left }}$, $\mathrm{L}$, and $\mathrm{V}_{\text {right }}$ are compared with the corresponding phase equilibrium obtained from the PeTS EOS 


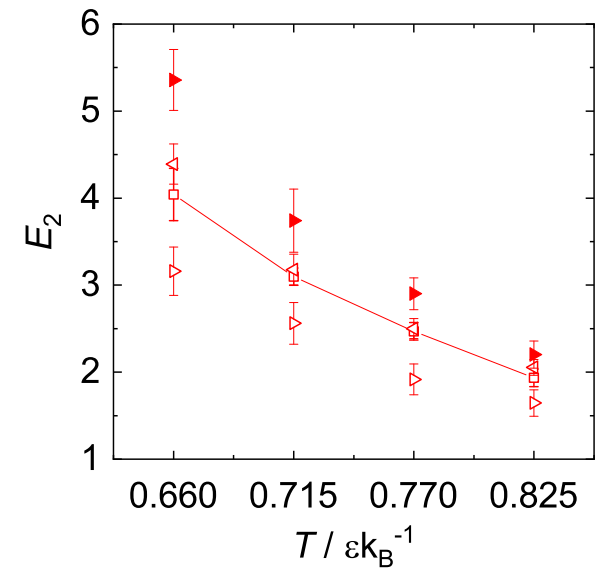

Figure 4. Enrichment of component $2 E_{2}$ at the interface at different temperatures during the mass transfer simulations (triangles) in comparison to the enrichment at equilibrium states (squares; adapted from Ref. [50]) at the same liquid phase composition. Left triangles and right triangles indicate the enrichment obtained for the left and right interface in the mass transfer simulations, respectively. The filled and empty right triangles indicate the enrichment using the vapour or liquid domain as reference, respectively (details are given in the text). Lines are a guide for the eye.

$[47,86]$ in the right panels of Fig. 3. The averaged state points sampled in the bulk domains agree astonishingly well with the isothermal phase envelope from the EOS. The individual state points in each bin for a bulk domain show an elliptic scattering around the averaged value, cf. Fig. A1 in the Appendix. The average pressure in the bulk domains decreases from left to right with the gradient of the chemical potential, as expected.

\subsection{Influence of the Temperature}

The influence of the temperature on the mass transfer in the two mixtures A and B was studied by performing additional simulations at three further temperatures $\left(T / \varepsilon k_{\mathrm{B}}^{-1}=0.66,0.77,0.825\right)$. The numeric values obtained for the mass fluxes and the non-equilibrium enrichment are reported in Table 1 and 2, respectively.

Density profiles and isothermal $p-x$ diagrams obtained during the quasi-stationary production phase for mixture $\mathrm{A}$ at $T / \varepsilon k_{\mathrm{B}}^{-1}=0.66,0.77,0.825$ are shown in Fig. 5. The corresponding plots for mixture B are presented in the Supplementary Material. For all four investigated temperatures (see section above), the difference of the chemical potential applied between the two control volumes was the same for mixture A and mixture B. The density profiles obtained for mixture A (cf. Fig. 5) show in all cases a gradient of the low-boiling component $\frac{\partial \rho_{2}}{\partial z}$, i.e. $\rho_{2}$ decreases from the left to the right side in each bulk domain. An enrichment of component 2 is observed in all cases for mixture A, but its height decreases with increasing temperature, which is in line with corresponding equilibrium results [50].

The enrichment computed from Eq. (5) for the left and right interface is depicted in Fig. 4 for all temperatures. As a reference, also the equilibrium enrichment at the corresponding temperature is shown [50]. As discussed above, the enrichment of the right interface was evaluated in two different 

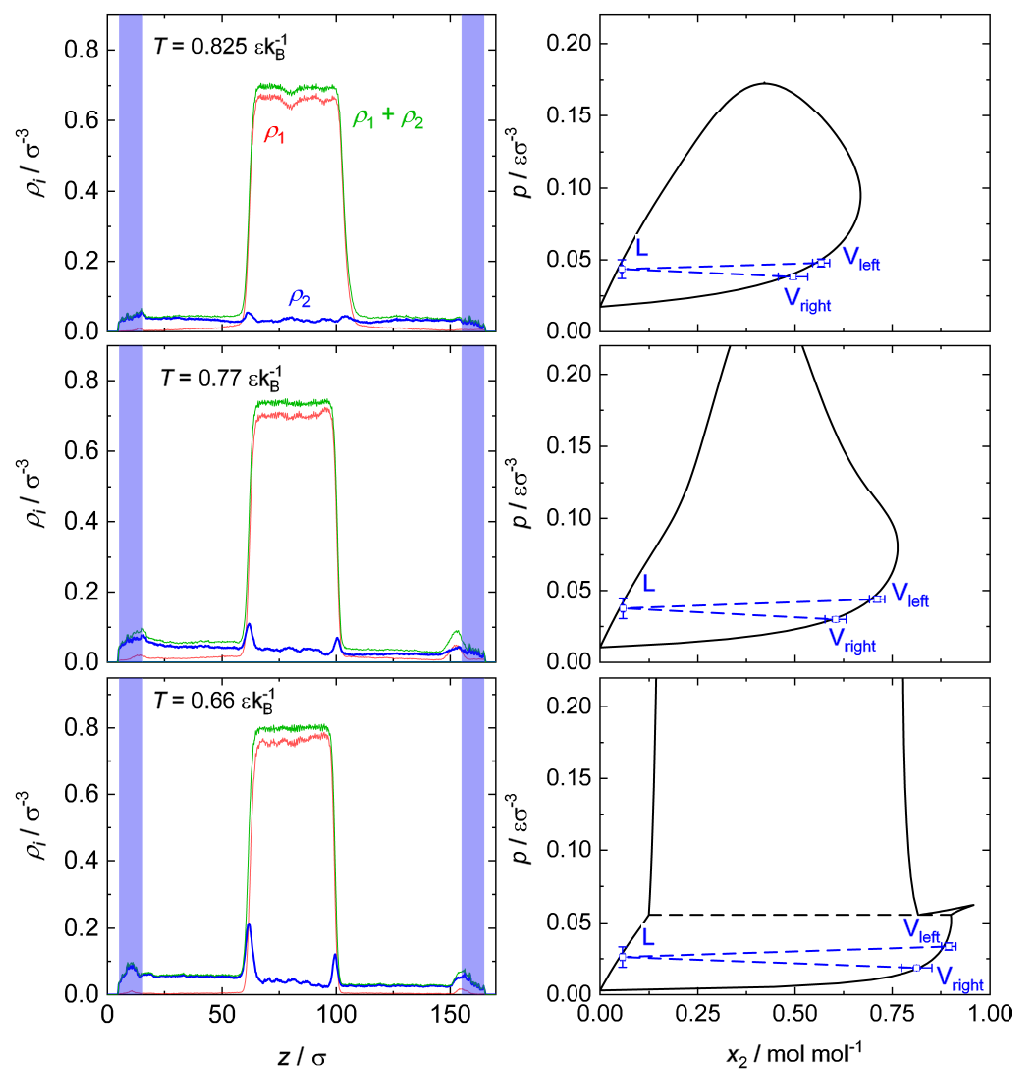

Figure 5. Density profiles (left panels) and isothermal $p-x$ diagrams (right panels) sampled during the production phase of the simulations of mixture A at different temperatures. The blue shaded area indicates the elongation of the control volumes in $z$-direction.

ways: using the averaged bulk liquid density (open triangles right) and using the vapour density (filled triangles right) for the evaluation of the denominator in Eq. (5). For all temperatures, the enrichment at the left interface is in fair agreement with the enrichment obtained from equilibrium simulations [50]. For the right interface, the evaluation based on the average liquid bulk density as reference, yields consistently lower enrichment than the equilibrium results. Vice versa, the evaluation based on the vapour bulk density reference yields larger enrichment. The differences between the enrichment obtained for the left and the right interface are likely due to the fact that interfacial properties strongly depend on the liquid bulk composition adjoined to the interface [48], which varies for the left and right interface in the mass transfer simulations. A comprehensive comparison of equilibrium and nonequilibrium interfacial properties is out of the scope of this work but is an interesting topic for future work.

In some of the density profiles shown in Fig. 5, small peaks of the total density in the vicinity of the control volumes indicate the presence of a small droplet. This is due to the fact, that the perturbations of the control volume act as nucleation precursor. Hence, in some cases, small nucleus form in the saturated vapour domains attached to the control volume. This also produces more pronounced fluctuations for the insertion rate than the removal rate of component 2 particles in the control volumes, cf. Supplementary Material. 
For all temperatures, the average state points computed for the three bulk domains $\mathrm{V}_{\text {left }}$, $\mathrm{L}$, and $\mathrm{V}_{\text {right }}$ are in excellent agreement with the corresponding phase equilibrium. The same holds for mixture B, see Supplementary Material. Also, the composition difference between the left and right bulk vapour domain is similar for the results from the different temperatures.

Fig. 6 shows the stationary mass flux $j_{2}$ sampled during the production phase as a function of the temperature for mixture A and B. For all studied temperatures, mixture B yields a significantly larger mass flux $j_{2}$ - especially at low temperatures. The mass flux obtained for mixture $\mathrm{B}$ exceeds that obtained for mixture A by a factor of two to four. This is astonishing as the diffusivities of both components are similar for both mixtures, both in the vapour and liquid phase (see Supplementary Material), the chemical potential gradient was the same and also the geometric parameters almost identical. The major difference is that mixture A shows a high enrichment while mixture B shows almost no enrichment. Hence, the present finding might point to an influence of the enrichment on the mass transfer. A further investigation of this hypothesis would require carrying out accompanying continuum simulations, which was not in the scope of the present work.

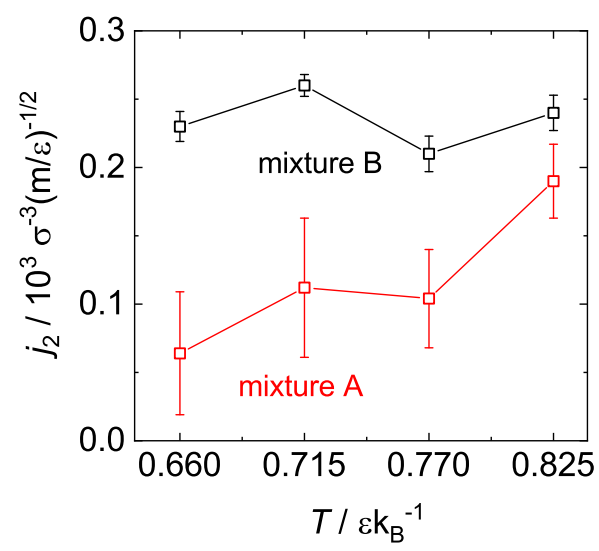

Figure 6. Mass flux $j_{2}$ sampled during the mass transfer simulations in mixture A and B at different temperatures. Lines are a guide for the eye.

\section{Conclusions}

The DCV method was applied for studying mass transfer in mixtures driven by a chemical potential difference across vapour-liquid interfaces by molecular simulations. It is based on a simulation scenario in which two vapour domains encase a liquid domain. Different values of the chemical potential of a selected component are prescribed in two control volumes in the two vapour domains and evoke a flux of that component that passes two vapour-liquid interfaces; the other components are stagnant due to the chosen boundary conditions. In isothermal simulations, the profiles of the component densities, the pressure tensor, and the flux of the selected component are measured. To the best of our knowledge, this 
is the first report on a molecular simulation method for studying mass transfer through vapour-liquid interfaces driven by chemical potential gradients. This is astonishing, as mass transfer through vapourliquid interfaces is very important in technical and natural processes. The new simulation scenario opens the way for detailed studies of these processes on the molecular level. In particular, the new simulation scenario can be used for systematic studies of the influence of mass transfer on interfacial properties, which are presently still lacking.

The simulation method was applied to study the influence of the enrichment of components at the vapour-liquid interface on the mass transfer. Two Lennard-Jones mixtures were examined, of which the phase equilibrium properties, including the interfacial equilibrium properties, are well-known [47, 48, 50]. The first mixture exhibits a large enrichment of the low-boiling component at the interface, whereas the second mixture exhibits no enrichment. We find that, even though both mixtures have similar bulk diffusivities and the same chemical potential gradient was applied, the mass flux observed in both systems differs significantly. These differences might be related to the presence of an enrichment at the interface in one of the mixtures. Hence, the results support the assumption [30, 47, 49, 69-72] that the enrichment influences the mass transfer through fluid interfaces. Further studies are, however, needed to prove or refute this hypothesis. The new scenario is a valuable tool for this. Such studies should also include simulations with continuum methods, that can be compared to the results from the molecular dynamics simulations. For such simulations, vapour and liquid bulk diffusivities are provided.

\section{Appendix A. Individual State Points in the Profiles}

Fig. A1 depicts a detailed comparison of the sampled state points from each bin (excluding the vicinity of the control volumes) from all profiles during the production phase from the mass transfer simulation with mixture A at $T=0.715 \varepsilon k_{\mathrm{B}}^{-1}$. The averaged state points for the two vapour domains and the liquid domain agree very well with the equilibrium phase envelope computed from the PeTS EOS $[47,86]$. The liquid bulk domain state points are connected to the two vapour domains by the state points in the interface. For the liquid bulk domain, the state points show an elliptic scatter pattern around a point on the phase equilibrium envelope. The scattering is dominated by a scattering in the pressure, which is simply due to the fact that the pressure shows stronger fluctuations in the liquid bulk than the vapour bulk, cf. the pressure profiles in Fig. 3. On the contrary, for vapour bulk domains $\mathrm{V}_{\text {left }}$ and $\mathrm{V}_{\text {right }}$, where the scattering of the individual bin state points is dominated by a scattering in the concentration. This is due to the fact that the relatively low number of particles in the vapour bins yields stronger fluctuations compared to the liquid bulk bins. Nevertheless, the three averaged state points from the bins in the respective bulk domain from all profiles during the production phase 
(blue squares in Fig. A1) are in excellent agreement with the phase equilibrium computed from the PeTS EOS [47, 86], which is known to give an excellent description of the phase equilibrium of LJTS mixtures [48, 50, 51].

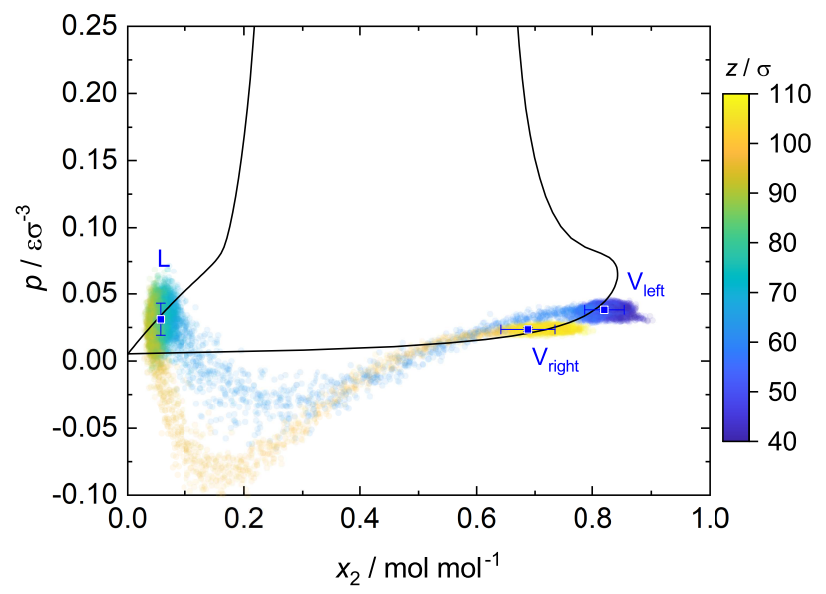

Figure A1. Pressure - composition diagram for mixture A at $T=0.715 \varepsilon k_{\mathrm{B}}^{-1}$. The black line indicates the phase equilibrium computed with the PeTS EOS $[47,86]$. The circles indicate state points sampled during the stationary phase of the simulation in the individual bins of the profiles - excluding the vicinity of the control volumes. The colour corresponds to the $z$-position in the profile. The squares indicate the averaged state points computed in each bulk domain (liquid as well as left and right vapour).

Following Ref. [96], the local scalar pressure $p$ in the heterogeneous system was simply computed by averaging the trace of the pressure tensor. The pressure across the interfaces smoothly connects the three bulk domains $\mathrm{V}_{\text {left }}-\mathrm{L}-\mathrm{V}_{\text {right }}$. The pressure transition in the two interfaces differs, which is in line with differences in the enrichment, cf. Fig. 4.

\section{Appendix B. Definition of Mass Fluxes and Reference Systems}

Velocities and fluxes reported in the main part of the paper refer to the fixed laboratory frame. The flux of component 2 is related to the mean directed velocity $u_{2}$ by

$$
j_{2}=\rho x_{2} u_{2} .
$$

Due to the chosen boundary conditions, the mean directed velocity of component 1 is zero here $\left(u_{1}=0\right)$, which results in a zero corresponding net flux of component $1\left(j_{1}=0\right)$. Hence, the diffusive and convective mass flux of component 1 cancel each other in the stationary state

$$
j_{1}^{\mathrm{D}}=-j_{1}^{\mathrm{conv}}
$$

The velocity $u_{2}$ is also the diffusion velocity of component 2 when the reference velocity is that of 
component 1.

$$
u_{2}^{\mathrm{D}, 1}=u_{2}
$$

However, often other reference velocities are used for defining the diffusive velocity and the corresponding diffusive fluxes, namely mole-averaging (or, equivalently particle number-averaging) and massaveraging. As the mass of all components is the same, there is no difference between these averages here. The mole-averaged velocity is:

$$
u_{0}^{\mathrm{n}}=x_{1} u_{1}+x_{2} u_{2}
$$

or, using $u_{1}=0$ :

$$
u_{0}^{\mathrm{n}}=x_{2} u_{2}
$$

Hence, using that reference velocity, the diffusion velocity of component $2 u_{2}^{\mathrm{D}, \mathrm{n}}$ is

$$
u_{2}^{\mathrm{D}, \mathrm{n}}=u_{2}-u_{0}=u_{2}-x_{2} u_{2}=x_{1} u_{2} .
$$

The corresponding diffusive flux is

$$
j_{2}^{\mathrm{D}, \mathrm{n}}=\rho x_{2} x_{1} u_{2}
$$

Comparing these results for the diffusion in the molar reference frame with the corresponding results in the fixed laboratory frame yields:

$$
u_{2}^{\mathrm{D}, \mathrm{n}} / u_{2}=j_{2}^{\mathrm{D}, \mathrm{n}} / j_{2}=x_{1}
$$

As the liquid bulk considered here consists mainly of the high-boiling component 1, the diffusive contributions (in the molar reference frame) are small in the liquid bulk. On the contrary, they are important in the vapour domain. 


\section{Acknowledgements}

The authors gratefully acknowledge funding of the present work by the ERC Advanced Grant ENRICO (grant agreement No. 694807). The simulations were carried out on the ELWE supercomputer at Regional University Computing Center Kaiserslautern (RHRK) under the grant TUK-MTD. The present research was conducted under the auspices of the Boltzmann-Zuse Society of Computational Molecular Engineering (BZS).

\section{Disclosure statement}

No potential conflict of interest was reported by the authors.

\section{Funding}

ERC Advanced Grant ENRICO [grant agreement No. 694807]. 


\section{References}

[1] Heinen M, Vrabec J, Fischer J. Communication: Evaporation: Influence of heat transport in the liquid on the interface temperature and the particle flux. The Journal of Chemical Physics. 2016;145(8):081101.

[2] Lotfi A, Vrabec J, Fischer J. Evaporation from a free liquid surface. International Journal of Heat and Mass Transfer. 2014;73:303.

[3] Chatwell RS, Heinen M, Vrabec J. Diffusion limited evaporation of a binary liquid film. International Journal of Heat and Mass Transfer. 2019;132:1296-1305.

[4] Heinen M, Vrabec J. Evaporation sampled by stationary molecular dynamics simulation. The Journal of Chemical Physics. 2019;151(4):044704.

[5] Tsuruta T, Tanaka H, Masuoka T. Condensation/evaporation coefficient and velocity distributions at liquidvapor interface. International Journal of Heat and Mass Transfer. 1999;42(22):4107-4116.

[6] Cheng S, Lechman JB, Plimpton SJ, Grest GS. Evaporation of Lennard-Jones fluids. The Journal of Chemical Physics. 2011;134(22):224704.

[7] Simon JM, Bedeaux D, Kjelstrup S, Xu J, Johannessen E. Interface film resistivities for heat and mass transfersintegral relations verified by non-equilibrium molecular dynamics. The Journal of Physical Chemistry B. 2006;110(37):18528-18536.

[8] Ge J, Bedeaux D, Simon J, Kjelstrup S. Integral relations, a simplified method to find interfacial resistivities for heat and mass transfer. Physica A: Statistical Mechanics and its Applications. 2007;385(2):421-432.

[9] Ge J, Kjelstrup S, Bedeaux D, Simon JM, Rousseau B. Transfer coefficients for evaporation of a system with a Lennard-Jones long-range spline potential. Physical Review E. 2007;75:061604.

[10] Matsumoto M. Molecular dynamics simulation of interphase transport at liquid surfaces. Fluid Phase Equilibria. 1996;125(1):195-203.

[11] Kryukov A, Levashov V. About evaporation-condensation coefficients on the vapor-liquid interface of high thermal conductivity matters. International Journal of Heat and Mass Transfer. 2011;54(13):3042 - 3048.

[12] Kon M, Kobayashi K, Watanabe M. Kinetic boundary condition in vapor-liquid two-phase system during unsteady net evaporation/condensation. European Journal of Mechanics - B/Fluids. 2017;64:81 - 92.

[13] Bird E, Liang Z. Transport phenomena in the Knudsen layer near an evaporating surface. Physical Review E. 2019;100:043108.

[14] Kjelstrup S, Hafskjold B. Nonequilibrium molecular dynamics simulations of steady-state heat and mass transport in distillation. Industrial \& Engineering Chemistry Research. 1996;35(11):4203-4213.

[15] Røsjorde A, Kjelstrup S, Bedeaux D, Hafskjold B. Nonequilibrium molecular dynamics simulations of steady-state heat and mass transport in condensation. II. transfer coefficients. Journal of Colloid and Interface Science. 2001;240(1):355-364.

[16] Røsjorde A, Fossmo D, Bedeaux D, Kjelstrup S, Hafskjold B. Nonequilibrium molecular dynamics simulations of steady-state heat and mass transport in condensation: I. local equilibrium. Journal of Colloid and Interface Science. 2000;232(1):178-185. 
[17] Inzoli I, Kjelstrup S, Bedeaux D, Simon J. Transfer coefficients for the liquid-vapor interface of a twocomponent mixture. Chemical Engineering Science. 2011;66(20):4533-4548.

[18] Hafskjold B, Ikeshoji T. Non equilibrium molecular dynamics simulation of coupled heat- and mass transport across a liquid/vapor interface. Molecular Simulation. 1996;16(1-3):139-150.

[19] van der Ham L, Bock R, Kjelstrup S. Modelling the coupled transfer of mass and thermal energy in the vapour-liquid region of a nitrogen-oxygen mixture. Chemical Engineering Science. 2010;65(6):2236-2248.

[20] Johannessen E, Gross J, Bedeaux D. Nonequilibrium thermodynamics of interfaces using classical density functional theory. The Journal of Chemical Physics. 2008;129(18):184703.

[21] Johannessen E, Bedeaux D. Integral relations for the heat and mass transfer resistivities of the liquid-vapor interface. Physica A: Statistical Mechanics and its Applications. 2006;370(2):258-274.

[22] Glavatskiy K, Bedeaux D. Transport of heat and mass in a two-phase mixture: From a continuous to a discontinuous description. The Journal of Chemical Physics. 2010;133(14):144709.

[23] Klink C, Waibel C, Gross J. Analysis of interfacial transport resistivities of pure components and mixtures based on density functional theory. Industrial \& Engineering Chemistry Research. 2015;54(45):11483-11492.

[24] Chilukoti HK, Kikugawa G, Ohara T. A molecular dynamics study on transport properties and structure at the liquid-vapor interfaces of alkanes. International Journal of Heat and Mass Transfer. 2013;59:144-154.

[25] Chilukoti HK, Kikugawa G, Ohara T. Structure and mass transport characteristics at the intrinsic liquidvapor interfaces of alkanes. The Journal of Physical Chemistry B. 2016;120(29):7207-7216.

[26] Chilukoti HK, Kikugawa G, Ohara T. Self-diffusion coefficient and structure of binary n-alkane mixtures at the liquid-vapor interfaces. The Journal of Physical Chemistry B. 2015;119(41):13177-13184.

[27] Duque D, Tarazona P, Chacon E. Diffusion at the liquid-vapor interface. The Journal of Chemical Physics. 2008;128(13):134704.

[28] Braga C, Muscatello J, Lau G, Müller EA, Jackson G. Nonequilibrium study of the intrinsic free-energy profile across a liquid-vapour interface. The Journal of Chemical Physics. 2016;144(4):044703.

[29] Liu P, Harder E, Berne BJ. On the calculation of diffusion coefficients in confined fluids and interfaces with an application to the liquid-vapor interface of water. The Journal of Physical Chemistry B. 2004; 108(21):6595-6602.

[30] Nagl R, Zimmermann P, Zeiner T. Interfacial mass transfer in water-toluene systems. Journal of Chemical \& Engineering Data. 2020;65(2):328-336.

[31] Braga C, Galindo A, Müller EA. Nonequilibrium molecular dynamics simulation of diffusion at the liquidliquid interface. The Journal of Chemical Physics. 2014;141(15):154101.

[32] Garrett BC, Schenter GK, Morita A. Molecular simulations of the transport of molecules across the liquid/vapor interface of water. Chemical Reviews. 2006;106(4):1355-1374.

[33] Baidakov VG, Protsenko SP. Molecular-dynamics simulation of relaxation processes at liquid-gas interfaces in single- and two-component lennard-jones systems. Colloid Journal. 2019 Sep;81(5):491-500.

[34] Baidakov VG, Protsenko SP, Bryukhanov VM. Relaxation processes at liquid-gas interfaces in one- and twocomponent Lennard-Jones systems: Molecular dynamics simulation. Fluid Phase Equilibria. 2019;481:1-14. 
[35] Lísal M, Brennan JK, Smith WR, Siperstein FR. Dual control cell reaction ensemble molecular dynamics: A method for simulations of reactions and adsorption in porous materials. The Journal of Chemical Physics. 2004;121(10):4901-4912.

[36] Arya G, Chang HC, Maginn EJ. A critical comparison of equilibrium, non-equilibrium and boundary-driven molecular dynamics techniques for studying transport in microporous materials. The Journal of Chemical Physics. 2001;115(17):8112-8124.

[37] Ható Z, Boda D, Kristóf T. Simulation of steady-state diffusion: Driving force ensured by dual control volumes or local equilibrium Monte Carlo. The Journal of Chemical Physics. 2012;137(5):054109.

[38] Martin MG, Thompson AP, Nenoff TM. Effect of pressure, membrane thickness, and placement of control volumes on the flux of methane through thin silicalite membranes: A dual control volume grand canonical molecular dynamics study. The Journal of Chemical Physics. 2001;114(16):7174-7181.

[39] Pohl P, Heffelfinger GS, Smith DM. Molecular dynamics computer simulation of gas permeation in thin silicalite membranes. Molecular Physics. 1996;89(6):1725-1731.

[40] Pohl PI, Heffelfinger GS. Massively parallel molecular dynamics simulation of gas permeation across porous silica membranes. Journal of Membrane Science. 1999;155(1):1-7.

[41] MacElroy JMD. Nonequilibrium molecular dynamics simulation of diffusion and flow in thin microporous membranes. The Journal of Chemical Physics. 1994;101(6):5274-5280.

[42] Sun L, Ely JF. Universal equation of state for engineering application: algorithm and application to nonpolar and polar fluids. Fluid Phase Equilibria. 2004;222-223:107-118.

[43] Heffelfinger GS, van Swol F. Diffusion in Lennard-Jones fluids using dual control volume grand canonical molecular dynamics simulation (DCV-GCMD). The Journal of Chemical Physics. 1994;100(10):7548-7552.

[44] Heffelfinger GS, Ford DM. Massively parallel dual control volume grand canonical molecular dynamics with LADERA I. gradient driven diffusion in Lennard-Jones fluids. Molecular Physics. 1998;94(4):659-671.

[45] Heffelfinger GS, Ford D. Massively parallel dual control volume grand canonical molecular dynamics with LADERA II. gradient driven diffusion through polymers. Molecular Physics. 1998;94(4):673-683.

[46] Stephan S, Hasse H. Enrichment at vapour-liquid interfaces of mixtures: Establishing a link between nanoscopic and macroscopic properties. International Reviews in Physical Chemistry. 2020;39(3):319-349.

[47] Stephan S, Langenbach K, Hasse H. Interfacial properties of binary Lennard-Jones mixtures by molecular simulations and density gradient theory. The Journal of Chemical Physics. 2019;150(17):174704.

[48] Stephan S, Hasse H. Molecular interactions at vapor-liquid interfaces: Binary mixtures of simple fluids. Physical Review E. 2020;101:012802.

[49] Stephan S, Langenbach K, Hasse H. Enrichment of components at vapour-liquid interfaces: A study by molecular simulation and density gradient theory. Chemical Engineering Transactions. 2018;69:295-300.

[50] Stephan S, Hasse H. Interfacial properties of binary mixtures of simple fluids and their relation to the phase diagram. Physical Chemistry Chemical Physics. 2020;22(22):12544-12564.

[51] Stephan S, Hasse H. Influence of dispersive long-range interactions on properties of vapour-liquid equilibria and interfaces of binary Lennard-Jones mixtures. Molecular Physics. 2020;118(9-10):e1699185. 
[52] Stephan S, Becker S, Langenbach K, Hasse H. Vapor-liquid interfacial properties of the binary system cyclohexane + CO2: Experiment, molecular simulation and density gradient theory. Fluid Phase Equilibria. 2020;518:112583.

[53] Becker S, Werth S, Horsch M, Langenbach K, Hasse H. Interfacial tension and adsorption in the binary system ethanol and carbon dioxide: Experiments, molecular simulation and density gradient theory. Fluid Phase Equilibria. 2016;427:476.

[54] Li W, Jin Z. Molecular dynamics simulation of natural gas-water interfacial tensions over wide range of pressures. Fuel. 2019;236:480-492.

[55] Fouad WA, Vega LF. The phase and interfacial properties of azeotropic refrigerants: the prediction of aneotropes from molecular theory. Physical Chemistry Chemical Physics. 2017;19:8977-8988.

[56] Niño-Amézquita OG, van Putten D, Enders S. Phase equilibrium and interfacial properties of water $+\mathrm{CO}_{2}$ mixtures. Fluid Phase Equilibria. 2012;332:40.

[57] Niño-Amézquita OG, Enders S. Phase equilibrium and interfacial properties of water + methane mixtures. Fluid Phase Equilibria. 2016;407:143-151.

[58] Llovell F, Dowell NM, Blas FJ, Galindo A, Jackson G. Application of the SAFT-VR density functional theory to the prediction of the interfacial properties of mixtures of relevance to reservoir engineering. Fluid Phase Equilibria. 2012;336(Supplement C):137-150.

[59] Enders S, Kahl H. Interfacial properties of water + alcohol mixtures. Fluid Phase Equilibria. 2008; 263(2):160-167.

[60] Chang TM, Dang LX. Liquid-vapor interface of methanol-water mixtures: A molecular dynamics study. The Journal of Physical Chemistry B. 2005;109(12):5759-5765.

[61] Cornelisse PM. The square gradient theory applied simultaneous modelling of interfacial tension and phase behaviour [dissertation]. Technische Universiteit Delft; 1997.

[62] Carey B. The gradient theory of fluid interfaces [dissertation]. Minneapolis: University of Minnesota; 1979.

[63] Falls AH, Scriven LE, Davis HT. Adsorption, structure, and stress in binary interfaces. The Journal of Chemical Physics. 1983;78(12):7300-7317.

[64] Mejía A, Cartes M, Segura H, Müller EA. Use of equations of state and coarse grained simulations to complement experiments: Describing the interfacial properties of carbon dioxide + decane and carbon dioxide + eicosane mixtures. Journal of Chemical \& Engineering Data. 2014;59(10):2928.

[65] Garrido JM, Cartes M, Mejía A. Coarse-grained theoretical modeling and molecular simulations of nitrogen + n-alkanes: (n-pentane, n-hexane, n-heptane, n-octane). The Journal of Supercritical Fluids. 2017;129:8390.

[66] Müller EA, Mejía A. Interfacial properties of selected binary mixtures containing n-alkanes. Fluid Phase Equilibria. 2009;282(2):68-81.

[67] Mejía A, Pàmies JC, Duque D, Segura H, Vega LF. Phase and interface behaviors in type-I and type-V Lennard-Jones mixtures: Theory and simulations. The Journal of Chemical Physics. 2005;123(3):034505.

[68] Miqueu C, Miguez JM, Piñeiro MM, Lafitte T, Mendiboure B. Simultaneous application of the gradient 
theory and Monte Carlo molecular simulation for the investigation of methane/water interfacial properties. The Journal of Physical Chemistry B. 2011;115(31):9618-9625.

[69] Klink C, Gross J. A density functional theory for vapor-liquid interfaces of mixtures using the perturbedchain polar statistical associating fluid theory equation of state. Industrial \& Engineering Chemistry Research. 2014;53(14):6169.

[70] Lee DJ, Telo da Gama MM, Gubbins KE. The vapour-liquid interface for a Lennard-Jones model of argonkrypton mixtures. Molecular Physics. 1984;53(5):1113-1130.

[71] Telo da Gama MM, Evans R. The structure and surface tension of the liquid-vapour interface near the upper critical end point of a binary mixture of Lennard-Jones fluids I. the two phase region. Molecular Physics. 1983;48(2):229-250.

[72] Garrido JM, Piñeiro MM, Mejía A, Blas FJ. Understanding the interfacial behavior in isopycnic LennardJones mixtures by computer simulations. Physical Chemistry Chemical Physics. 2016;18:1114-1124.

[73] Karlsson B, Friedman R. Dilution of whisky - the molecular perspective. Scientific Reports. 2017;7(6489):19.

[74] Cahn JW, Hilliard JE. Free energy of a nonuniform system. I. interfacial free energy. The Journal of Chemical Physics. 1958;28(2):258.

[75] Cahn JW. On spinodal decomposition. Acta Metallurgica. 1961;9(9):795-801.

[76] Stephan S, Thol M, Vrabec J, Hasse H. Thermophysical properties of the Lennard-Jones fluid: Database and data assessment. Journal of Chemical Information and Modeling. 2019;59(10):4248-4265.

[77] Stephan S, Staubach J, Hasse H. Review and comparison of equations of state for the Lennard-Jones fluid. Fluid Phase Equilibria. 2020;523:112772.

[78] Stephan S, Dyga M, Urbassek H, Hasse H. The influence of lubrication and the solid-fluid interaction on thermodynamic properties in a nanoscopic scratching process. Langmuir. 2019;35(51):16948-16960.

[79] Horsch M, Hasse H, Shchekin AK, Agarwal A, Eckelsbach S, Vrabec J, Müller EA, Jackson G. Excess equimolar radius of liquid drops. Physical Review E. 2012;85(3):031605.

[80] Eckhardt W, Heinecke A, Bader R, Brehm M, Hammer N, Huber H, Kleinhenz HG, Vrabec J, Hasse H, Horsch M, Bernreuther M, Glass CW, Niethammer C, Bode A, Bungartz HJ. 591 tflops multi-trillion particles simulation on SuperMUC. In: Supercomputing; Berlin, Heidelberg. Springer; 2013. p. 1-12.

[81] Stephan S, Liu J, Langenbach K, Chapman WG, Hasse H. Vapor-liquid interface of the Lennard-Jones truncated and shifted fluid: Comparison of molecular simulation, density gradient theory, and density functional theory. The Journal of Physical Chemistry C. 2018;122(43):24705-24715.

[82] Stephan S, Horsch M, Vrabec J, Hasse H. MolMod - an open access database of force fields for molecular simulations of fluids. Molecular Simulation. 2019;45(10):806-814.

[83] Allen MP, Tildesley DJ. Computer simulation of liquids. Oxford: Oxford University Press; 1989.

[84] Lorentz HA. Über die Anwendung des Satzes vom Virial in der kinetischen Theorie der Gase. Annalen Der Physik. 1881;248:127-136.

[85] Berthelot D. Sur le mélange des gaz. Comptes rendus hebdomadaires des séances de 1'Académie des Sciences. 
1898;126:1703-1706.

[86] Heier M, Stephan S, Liu J, Chapman WG, Hasse H, Langenbach K. Equation of state for the Lennard-Jones truncated and shifted fluid with a cut-off radius of $2.5 \sigma$ based on perturbation theory and its applications to interfacial thermodynamics. Molecular Physics. 2018;116(15):2083-2094.

[87] Taylor R, Krishna R. Multicomponent mass transfer. New York: John Wiley \& Sons; 1993.

[88] Niethammer C, Becker S, Bernreuther M, Buchholz M, Eckhardt W, Heinecke A, Werth S, Bungartz HJ, Glass CW, Hasse H, Vrabec J, Horsch M. ls1 mardyn: The massively parallel molecular dynamics code for large systems. Journal of Chemical Theory Computation. 2014;10(10):4455.

[89] Poling BE, Prausnitz JM, O’Connell JP. The properties of gases and liquids. 5th ed. New York: McGrawHill; 2001.

[90] Rutkai G, Köster A, Guevara-Carrion G, Janzen T, Schappals M, Glass CW, Bernreuther M, Wafai A, Stephan S, Kohns M, Reiser S, Deublein S, Horsch M, Hasse H, Vrabec J. ms2: A molecular simulation tool for thermodynamic properties, release 3.0. Computer Physics Communications. 2017;221:343-351.

[91] Fernandez GA, Vrabec J, Hasse H. Self diffusion and binary Maxwell-Stefan diffusion in simple fluids with the Green-Kubo method. International Journal of Thermophysics. 2004;25(1):175-186.

[92] Fernández GA, Vrabec J, Hasse H. Self-diffusion and binary Maxwell-Stefan diffusion coefficients of quadrupolar real fluids from molecular simulation. International Journal of Thermophysics. 2005;26:13891407.

[93] Guevara-Carrion G, Vrabec J, Hasse H. Prediction of self-diffusion coefficient and shear viscosity of water and its binary mixtures with methanol and ethanol by molecular simulation. The Journal of Chemical Physics. 2011;134(7):074508.

[94] Bellaire D, Kiepfer H, Münnemann K, Hasse H. PFG-NMR and MD simulation study of self-diffusion coefficients of binary and ternary mixtures containing cyclohexane, ethanol, acetone, and toluene. Journal of Chemical \& Engineering Data. 2020;65(2):793-803.

[95] Par̆ez S, Guevara-Carrion G, Hasse H, Vrabec J. Mutual diffusion in the ternary mixture of water + methanol + ethanol and its binary subsystems. Phys Chem Chem Phys. 2013;15:3985-4001.

[96] Imre AR, Mayer G, Házi G, Rozas R, Kraska T. Estimation of the liquid-vapor spinodal from interfacial properties obtained from molecular dynamics and lattice boltzmann simulations. The Journal of Chemical Physics. 2008;128(11):114708. 